\title{
Self-consistent Modelling of the interstellar medium*
}

\author{
Dieter Breitschwerdt \\ Max-Planck-Institut für extraterrestrische Physik, Postfach 1312, D-85741 \\ Garching, Germany; Email: breitsch@mpe.mpg.de
}

November 30, 2002

\begin{abstract}
The dynamical evolution of hot optically thin plasmas in the ISM crucially depends on the heating and cooling processes. It is essential to realize that all physical processes that contribute operate on different time scales. In particular detailed balancing is often violated since the statistically inverse process of e.g. collisional ionization is recombination of an ion with two electrons, which as a threebody collision is usually dominated by radiative recombination, causing a departure from collisional ionization equilibrium. On top of these differences in atomic time scales, hot plasmas are often in a dynamical state, thereby introducing another time scale, which can be the shortest one.

The non-equilibrium effects will be illustrated and discussed in the case of galactic outflows. It will be shown, that spectral analyses of X-ray data of edge-on galaxies show a clear signature in the form of "multi-temperature" halos, which can most naturally be explained by the "freezing-in" of highly ionized species in the outflow, which contribute to the overall spectrum by delayed recombination. This naturally leads to a non-equilibrium cooling function, which modifies the dynamics, which in turn changes the plasma densities and thermal energy budget, thus feeding back on the ionization structure. Therefore self-consistent modelling is needed.
\end{abstract}

Keywords: ISM: general, galaxies: halos, galaxies: starburst, X-rays: galaxies

\section{Introduction}

Interstellar plasmas are subject to numerous dynamical and thermal processes, which are essentially driven by star formation activity in galaxies. Matter and radiation are continuously exchanged between the interstellar medium (ISM) and stars. This can loosely be described as a matter cycle. Star formation induced by gravitational instabilities is only possible if both transport of angular momentum, mass and energy work efficiently, with radiative cooling playing a key rôle both in the formation of interstellar clouds and protostars. During its main sequence lifetime a star interacts with the ISM by its constant photon output, by which especially Lyman-continuum photons can inject a substantial

* XMM-Newton is an ESA Science Mission with instruments and contributions directly funded by ESA Member states and the USA (NASA). The XMM-Newton project is supported by the Bundesministerium fr Bildung und Forschung/Deutsches Zentrum für Luft- und Raumfahrt (BMBF/DLR), the Max-Planck-Gesellschaft and the Heidenhain-Stiftung.

(C) 2018 Kluwer Academic Publishers. Printed in the Netherlands. 
amount of energy creating HII regions. Moreover mass loss in the form of stellar winds gives rise to hot shock heated extended bubbles due to a mechanical wind luminosity of the order of $10^{36} \mathrm{erg} / \mathrm{s}$, corresponding to about $30 \%$ of the energy of a type II supernova (SN) integrated over the stellar main sequence life time. However, the most efficient energy and momentum input into the ISM is by SN explosions, both randomly or in concert thus generating superbubbles. Shock velocities are initially of the order of $10^{3} \mathrm{~km} / \mathrm{s}$, and therefore from simple considerations of Rankine-Hugoniot conditions for strong shocks and adiabatic coefficient $\gamma=5 / 3$, the post shock temperature obeys the simple relation: $T_{s h} \approx \frac{3}{16}\left(\mu \bar{m} / k_{B}\right) v_{8}^{2} \approx 2.7 \times 10^{7} \mu v_{8}^{2} \mathrm{~K}$, with $\mu, \bar{m}, k_{B}$ and $v_{8}$ being the mean molecular weight, mean particle mass, Boltzmann's constant and the upstream shock velocity (in the shock rest frame) in units of $1000 \mathrm{~km} / \mathrm{s}$, respectively. Thus radiative cooling will be by thermal bremsstrahlung, recombination lines as well as atomic line transitions, detectable mainly in soft X-rays. It should be emphasized however, that the bulk of the energy is lost by adiabatic expansion of the supernova remnant (SNR) or superbubble (SB). This can be attributed to the fact that there exists a huge overpressure with respect to the ambient medium, acting like a piston, and that the radiative cooling time of low density bubbles is usually much larger than the timescale by which they merge with the ISM. As a rough estimate, we can compare the energy loss rate per unit volume with the $p d V$-work done by the expanding bubble: $\Xi=n^{2} \Lambda(T) t /\left(2 n k_{B} T\right) \approx 1.1 \times 10^{7}(n / T) t_{6}<1$, as long as $T \gtrsim 10^{6} \mathrm{~K}$, using $\Lambda(T) \lesssim 10^{-22} \mathrm{erg} \mathrm{cm}^{3} / \mathrm{s}$ and $t_{6}$ being the SB lifetime in units of Myr, where $T$ and $n$ are the plasma temperature and density, respectively. Note that during the adiabatic stage of evolution, similarity solutions may be applied, thus $n \propto t^{\alpha}$, with $\alpha=-4 / 5$ for SBs and $\alpha=-6 / 5$ for SNRs. For $T \sim$ const. during the energy driven phase this implies $\Xi \propto t^{1 / 5}$ for SBs and $\Xi \propto t^{-1 / 5}$ for SNRs. Thus if $\Xi(t) \ll 1$ initially, it will be so later on due to the weak time dependence of $\Xi$. For $n<10^{-3} \mathrm{~cm}^{-3}, T>10^{6} \mathrm{~K}$, as it is expected for superbubbles, this will definitely hold, except, maybe, for bubbles in very dense regions.

On a larger scale, where star forming regions (SFRs) are clustered, the combined pressure of winds and SN explosions can drive a galactic fountain or wind. Such a flow is again characterized by strong adiabatic and radiative cooling. Most prominent examples are starburst galaxies, from which so-called "superwinds" emanate (e.g. Heckman et al., 1990). Common to all examples mentioned here is a coupling between the thermal and the dynamical evolution, which will be described and discussed in the following sections. 


\section{Non-equilibrium cooling of interstellar plasmas}

Interstellar cooling is on a fundamental level a complicated process. The energy loss rate per unit volume, expressed as the cooling function, $\Lambda(T, Z)$, when normalized to the electron and ion densities, not only depends on temperature $T$ and the elemental abundances $Z$ of the fluid, but in general also on the distribution of ionization stages. The assumption of collisional ionization equilibrium (CIE) circumvents this problem. It is assumed that the rate of ionization by inelastic collions between electrons and ions is exactly balanced by the rate of recombinations. While the former is a cooling process the latter as a statistically inverse process must consequently be a heating process. Since the energy released has to be transferred to a third particle, thereby increasing the thermal energy of the plasma, such three-body interactions are extremely improbable in a tenuous, hot optically thin plasma like in a bubble or a superwind. Therefore it is commonly taken that radiative recombination is dominating; however, this is also a cooling process to the plasma. Thus a hot plasma, left to itself will eventually depart from equilibrium (s. Shapiro \& Moore, 1976; Schmutzler \& Tscharnuter, 1993).

The situation can be more dramatic, if the plasma undergoes compression like e.g. in a shock wave, or expansion like in a SB, fountain or wind. The first case gives rise to so-called underionized plasmas: the electrons downstream of a collisionless shock are heated up very efficiently by randomizing their kinetic energy via turbulent electromagnetic fields, and on a somewhat larger timescale also the ions. The ionization process on the other hand depends on the cross sections and thus on atomic timescales, which are much larger. Therefore the ionization will lag behind, and when observed spectroscopically, the plasma will appear cooler than it actually is (see e.g. Cox \& Anderson, 1982). The second case has the opposite characteristics, and is known as an overionized plasma: fast adiabatic expansion, like e.g. in an expanding wind, which occurs on much shorter timescales than radiative recombination, makes the plasma appear hotter to the observer due to delayed recombination (see Breitschwerdt \& Schmutzler, 1994). It has been shown that in these non-equilibrium ionization (NEI) cases the dynamics of the plasma and its thermal evolution are closely intertwined. The dynamics changes the density and temperature, which in turn changes the ionization structure, which determines the cooling function, which again modifies the dynamics. Thus a self-consistent description is necessary. Under CIE assumptions, the distribution of ionization stages is a sensitive function of temperature and thus the measurement of line ratios (e.g. NV/OVI) leads to a reliable tempera- 


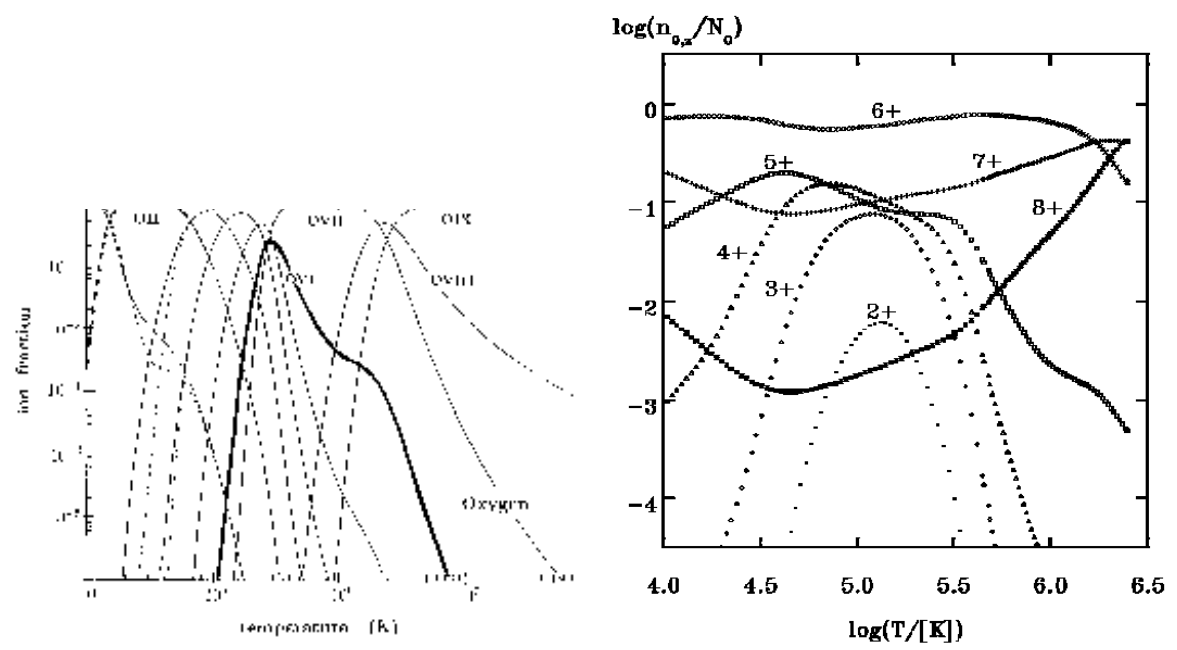

Figure 1. Left: Ionization fractions for oxygen as a function of temperature (taken from Böhringer and Hensler, 1989) for a plasma in collisional ionization equilibrium (CIE). Right: Ionization fractions for oxygen as a function of temperature for a plasma in non-equilibrium (NEI) taken from Breitschwerdt and Schmutzler (1999).

ture determination; however, in the case of NEI this is no longer valid (s. Fig. 1). In essence the plasma keeps a memory of its origin, and due to fast adiabatic cooling a particular ion can be present over a wide range of temperatures. Therefore when modelling the emissivity and the spectrum of a plasma, the time-dependent evolution of the ionization stages has to be followed closely along with the evolution of the astrophysical model (s. Fig. 2). Before turning to some detailed examples, some caveats for using plasma models have to be pointed out: although there exists a large data base of atomic cross sections for the various processes included, such as collisional and autoionization, photo-ionization, radiative and dielectronic recombination, two-photon emission, Auger effect, charge exchange reactions, just to name the most important ones, there are still quantitative discrepancies in plasma codes on the market (cf. Masai, 1997), particularly for heavy elements like iron around $1 \mathrm{keV}$ (up to a factor of 2 in emissivity). Moreover, when fitting observed X-ray spectra it is strictly speaking wrong to substitute a CIE fit model (e.g. RS, MEKAL etc.) by a "nonequilibrium model" that has just one or more free parameters, like e.g. the ionization parameter $I=n_{e} t$, simply because it is not calculated self-consistently with the dynamical evolution. The evolution of the astrophysical model is a necessary although not sufficient input. It cannot even be claimed that such a "NEI fit model is closer to the 


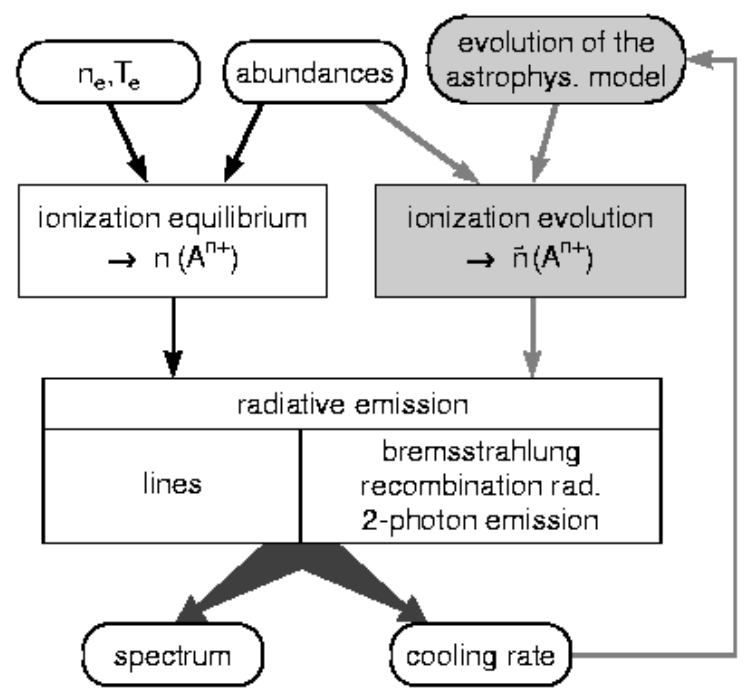

Figure 2. Flow chart of plasma emission model (s. Böhringer, 1997). White boxes refer to equilibrium (CIE), grey boxes to additions necessary for non-equilibrium (NEI) models. $A^{n+}$ denotes atomic species $A$ ionized n-times.

truth than the CIE model, if it cannot be physically motivated; it just fits better due to a larger number of free parameters.

\section{Spectral signatures of galactic outflows}

In the following it will be demonstrated that NEI cooling leaves a distinct imprint on the plasma, which can already be seen by current soft X-ray observations, even at modest spectral resolutions. The reason being the temperature sensitivity of CIE spectra and the existence of diagnostic ions, such as lithium and helium like oxygen and iron L shell transitions. We have analyzed $23 \mathrm{ksec}$ of ROSAT PSPC archival data in the $0.1-2.4 \mathrm{keV}$ range of the nearby edge-on galaxy NGC 253 (Breitschwerdt \& Freyberg, 2003) retaining $15.3 \mathrm{ksec}$ of good background subtracted data (s. Fig. 3) and obtained only a statistically acceptable fit by using two CIE plasma temperatures (applying the plasma code of Raymond \& Smith, 1977 (RS)); alternatively decreasing the elemental abundances to about $0.05 Z_{\odot}$ was equally satisfactory. However, it seems rather odd, that a SN heated and thus chemically enriched superwind should have highly subsolar abundances (although this has been claimed in the literature). On the other hand, we have simulated the outflow by a galactic wind model (for details s. Breitschwerdt 

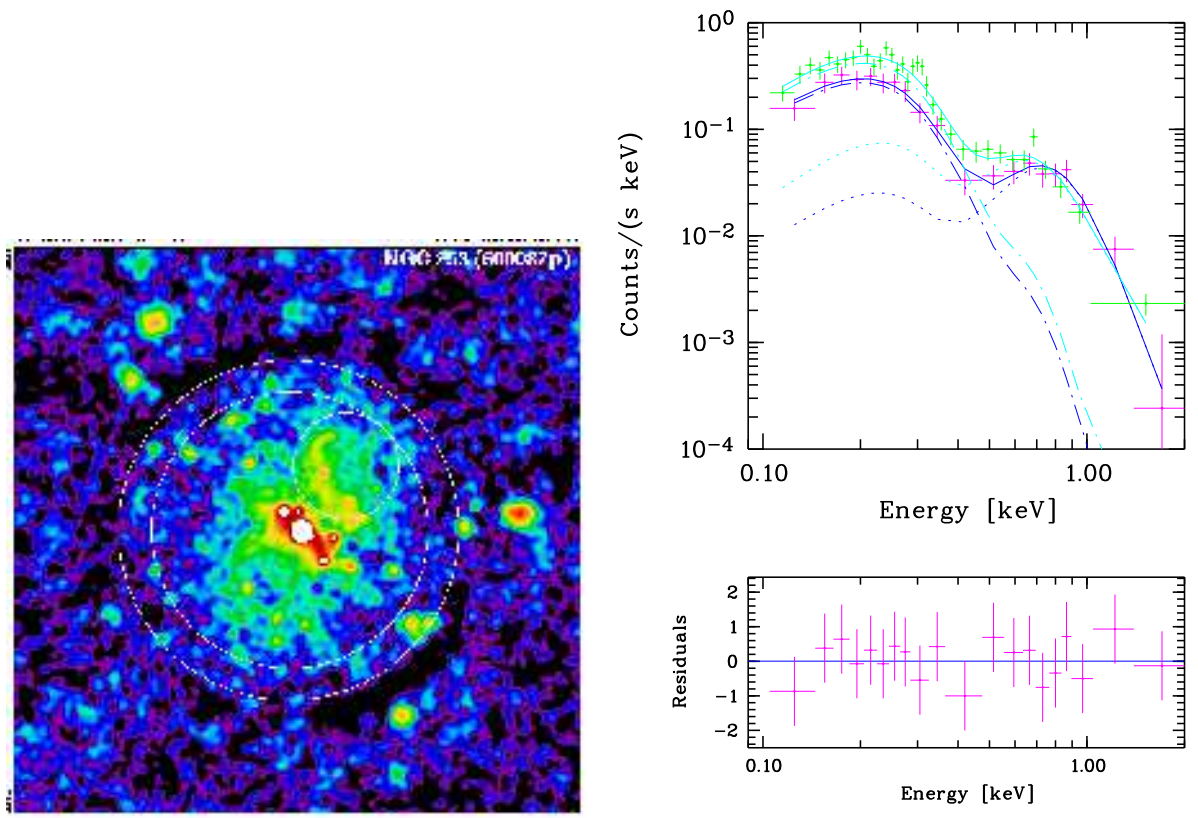

Figure 3. Left: ROSAT PSPC observation of the edge-on starburst galaxy NGC 253 (north is up) in soft X-rays $(0.1-2.4 \mathrm{keV})$ smoothed with a Gaussian filter; colour coding is from blue or dark (low) to red or bright (high intensity). The small circle shows the halo region that has been analyzed, while the outer ring represents the region that has been used for background subtraction. Right: Comparison between data (red points with error bars, lower curve) and self-consistent model M4 (green points, upper curve). The solid, dashed, dash-dotted and dotted curves are plasma fit models (2 Raymond \& Smith (1977) CIE models) with solar abundances, respectively). There is also good quantitative agreement between data and model, when taking into account missing flux in the observations due to point source masking.

\& Schmutzler, 1999) calculating self-consistently the full NEI plasma structure as discussed before. The resulting spectrum, integrated over the field of view (FOV) of the PSPC, binned into PSPC channels and folded through the instrumental response, was both quantitatively and qualitatively similar to the observed one (s. Fig. 3). We have thus treated the observed and the simulated spectra in exactly the same manner. Moreover, spectral fitting of the model data was again satisfactory for $2 \mathrm{RS}$ plasmas or abundances of $Z \approx 0.05 Z_{\odot}$. Hence it is fair to conclude that highly subsolar abundances are an artefact of the fit procedure. Clearly decreasing the abundances, washes out the prominent and characteristic lines of a spectrum and therefore gives more flexibility to accommodate all the temperature sensitive lines in a CIE spectrum! The boundary conditions of the outflow model reflect the SFR in the underlying galactic disk, but are specified at 
Em. Sp. of NGC 253 (M1) at $\mathrm{T}=2.50069 \times 10^{5}[\mathrm{~K}](\mathrm{NEI}+\mathrm{CIE})$

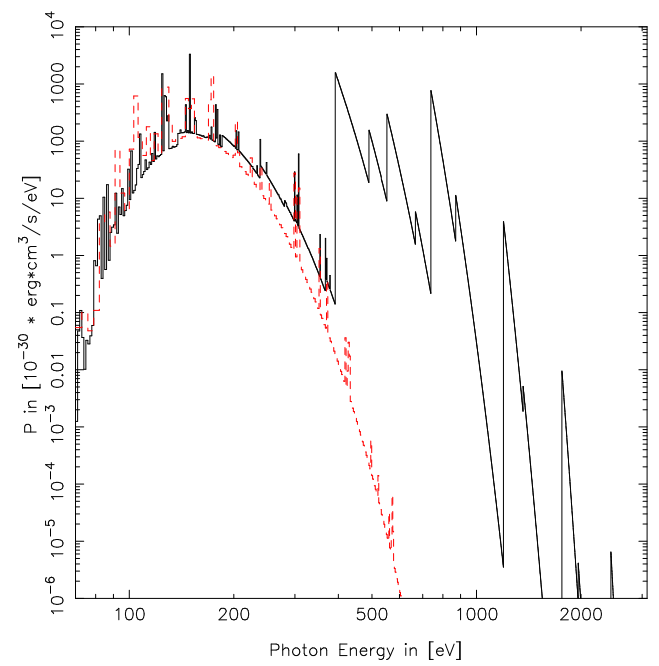

Cooling functions (Equil. RS vs. NEI 1st it.) NGC3079 (M5)

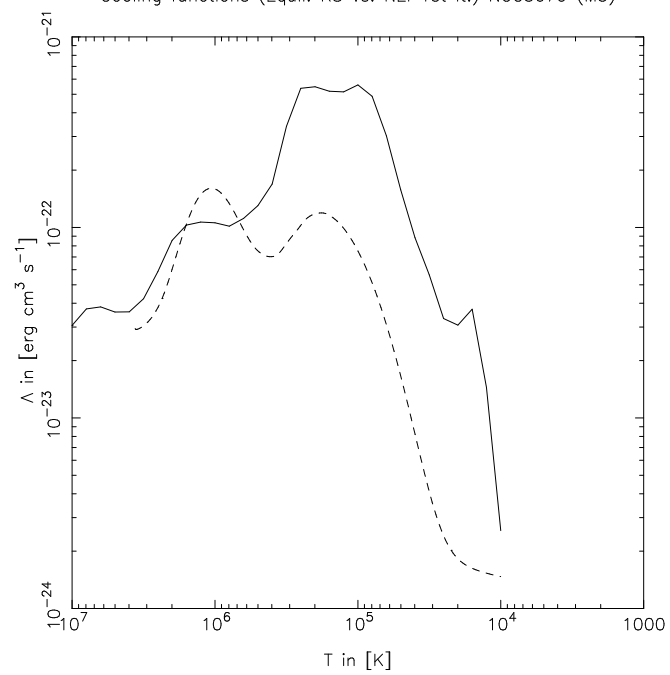

Figure 4. Left: Comparison of a CIE and a NEI emission spectrum resulting from a simulated outflow of the starburst galaxy NGC 253 at a distance (and time) when the temperature has cooled down $2.5 \times 10^{5} \mathrm{~K}$. The characteristic saw-tooth structure is due to recombination lines, here of SiXII and FeXVII (two main peaks, with energy increasing). Right: Comparison of CIE (solid curve) cooling function $\Lambda$ and the one resulting from a self-consistent evolution (dashed curve) of ionization stages for the successful spectral fit model of the halo emission of NGC 3079.

a vertical distance of $z=1 \mathrm{kpc}$ at the base of the halo. The flow geometry is represented by a flux tube, following the transition from planar close to the disk to spherically divergent flow at large distances. The gravitational potential has been described by (oblate) spheroids, including a bulge, a disk and a dark matter halo component (to account for the flatness of the rotation curve), scaled to observations. For the successful model, hot plasma is injected at the base of the halo at a temperature $T=2.5 \times 10^{6} \mathrm{~K}$ and a density $n=7 \times 10^{-3} \mathrm{~cm}^{-3}$. It is noteworthy that the models are fairly well constrained, even by the PSPC spectral resolution. The intrinsic outflow spectra, corresponding to disk parallel cuts through the halo at a given distance from the galactic plane, are radically different from CIE spectra (s. Fig. 3), characterized by strong saw-tooth type recombination lines due to delayed recombination. Note that both the elements and the ionization stages of the most intense lines change as a function of time (or distance from the disk), characterized by their recombination time scale. Another marked difference between CIE and NEI spectra is the existence of high energy X-ray photons in the overionized case (at around $1 \mathrm{keV}$ in Fig. 3), although the temperature is already down to $2.5 \times 10^{5} \mathrm{~K}$. This occurs, because the plasma was once hot enough to have generated 

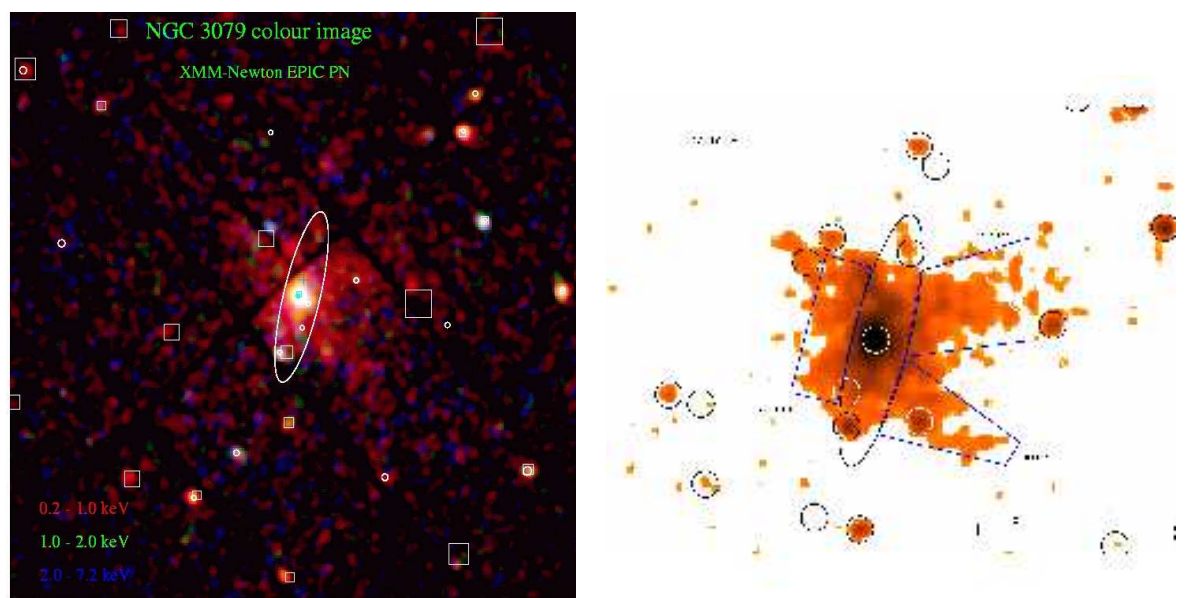

Figure 5. Left: RGB image of the extended X-ray halo of the nearby edge-on starburst galaxy NGC 3079, as observed by the XMM-Newton EPIC pn camera. Right: Smoothed image of the diffuse emission of NGC 3079 in the $0.2-1.0 \mathrm{keV}$ energy band (Breitschwerdt et al. 2003). Point sources are shown by circles. The $D_{25}$ ellipse is indicated to show the optical extension of the disk.

these photons in the past: the plasma retains a memory of its origin. The integrated spectrum, which is folded through the detector response matrix for comparison with the observed data (s. Fig. 3), is the sum of such intrinsic spectra over the FOV.

Another striking example of an overionized outflow is the LINER edge-on starburst galaxy NGC 3079, which we have observed with XMMNewton for $25 \mathrm{ksec}$ (Breitschwerdt et al. 2003). The image taken with the EPIC pn camera shows an extended soft X-ray halo (especially impressive in the $0.2-1 \mathrm{keV}$ band) of roughly the same size as the $D_{25}$-ellipse, representing the optical diameter of the disk (s. Fig. 3). It can be clearly seen that apart from the nuclear outflow, there also exists an extended component along the major and minor axes. Its spiky morphology is probably related to the SFR in the underlying disk. We have analyzed and fitted the EPIC pn spectrum of the NE spur (Breitschwerdt et al., 2003). The data are shown as crosses (error bars) and clearly exhibit two humps, which we identify as due to oxygen (at around $0.5 \mathrm{keV}$ ) and iron $\mathrm{L}$ shell line $(0.8-0.9 \mathrm{keV})$ complexes. This is corroborated by running again an outflow simulation, generating a self-consistent NEI spectrum and subjecting it to the same procedure as before (i.e. folding through the EPIC pn instrumental response). The best model fit (with a reduced $\chi^{2} \approx 1.2$ ) requires a temperature of $T=3.56 \times 10^{6} \mathrm{~K}$ and a density of $n=5 \times 10^{-3} \mathrm{~cm}^{-3}$ at the base of the halo. The outflow velocity there is about $220 \mathrm{~km} / \mathrm{s}$, reaching about $450 \mathrm{~km} / \mathrm{s}$ at $z=50 \mathrm{kpc}$. The higher initial temperature as 

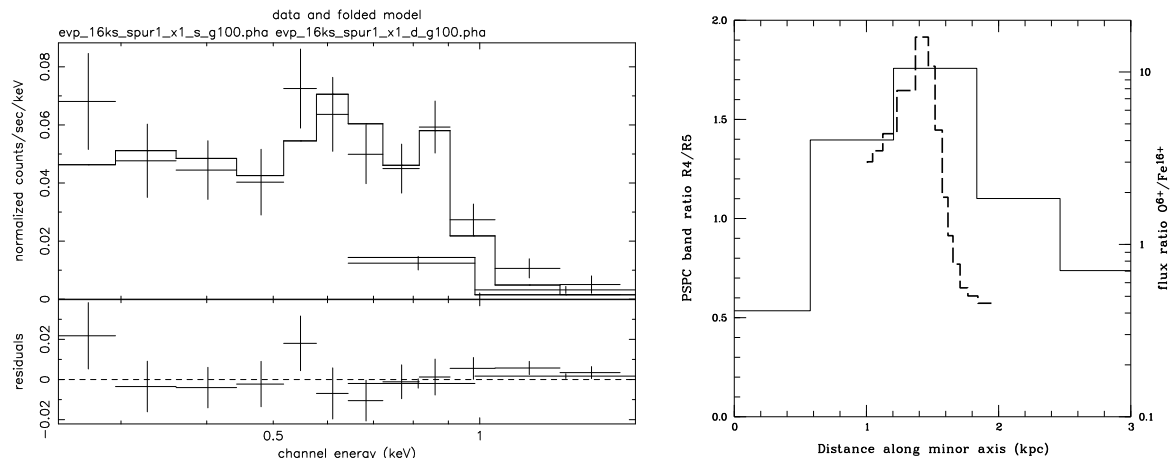

Figure 6. Left: Fit (solid line: $\chi^{2}=1.2$ ) of the XMM-Newton Epic pn observed spectrum (crosses correspond to error bars) of the emission of NGC 3079 (0.2 - 2 $\mathrm{keV}$ ) by a self-consistent NEI outflow model (solid line; Breitschwerdt et al. 2003); the lower panel shows the fit residuals. Right: PSPC R4 $(520-690 \mathrm{eV})$ and R5 $(700-900 \mathrm{eV})$ band ratio in a disk parallel stripe $(\Delta z=2 \mathrm{kpc})$ along the minor axis of NGC 253 (solid histogram) compared to OVII /FeXVII flux ratio averaged over $625-675 \mathrm{eV}$ and $805-865 \mathrm{eV}$, respectively (dashed histogram), derived from the best fit halo model, according to self-consistent NEI calculations (cf. Fig. 3).

compared to NGC 253 reflects the more violent outflow in this case and is necessary for producing the iron bump. We find again that no single RS model can fit the spectrum. The discrepancy between the cooling rates in CIE and NEI can be quite substantial at certain temperatures and thus have a pronounced effect on the outflow dynamics, as can be seen by comparing the respective cooling curves in Fig. 3: delayed recombination can indeed reduce the cooling rate.

To go one step further, if photon statistics of the observational data permits, one can analyze the spectral behaviour of the plasma in disk parallel stripes of widths of $\Delta z \sim 2 \mathrm{kpc}$ using a "sliding window" technique (s. Fig. 3). The idea is to search for "diagnostic ions" of starburst driven outflows. The double hump in Fig. 3 suggests OVII and FeXVII as spectral signatures that should vary in strength as the outflow cools down adiabatically and radiatively. We have therefore compared the PSPC R4 $(520-690 \mathrm{eV})$ to R5 $(700-900 \mathrm{eV})$ band ratios along the minor axis to an averaged OVII $(625-675) \mathrm{eV}$ to FeXVII $(805-865 \mathrm{eV})$ ratio of the model calculations. Although the latter ones have arbitrarily high "spectral resolution", we have averaged over an energy range free of other spectral features; hence the energy range is smaller than in the corresponding ROSAT PSPC bands, and the absolute fluxes do not match. However, it can be seen that the flux ratio of these characteristic ions have pronounced peak at about 1.5 kpc from the disk both for the observed and the model spectra. This gives a good hint that the time-dependent evolution of these ion species 
in the outflow should be basically correct. More stringent conclusions have to be deferred to an analysis of higher resolution spectra.

\section{Conclusions}

The discrepancy between equilibrium plasma models and observational data in the EUV and soft X-rays is commonly felt as a nuisance. There exists e.g. a plethora of high spectral resolution data for the Local Bubble. It is indeed embarrassing that presently neither SNR nor SB models, with CIE or simple NEI cooling can reproduce all these data satisfactorily. However, I would like to stress a positive aspect of this problem: the spectral fingerprints obtained contain far more information than our simple models can handle. Although being intrinsically more complicated, the advantage of NEI models is to connect the spectrum of a plasma at any given time to its history and therefore to help us to learn something about the plasma's excitation conditions and hence its origin. In case of the Local Bubble it seems that more sophisticated 3D simulations including NEI cooling, as undertaken currently (Avillez \& Breitschwerdt, 2003), should lead to an improvement.

Meanwhile it is worth focusing also on somewhat simpler astrophysical problems such as galactic outflows, which are well observable in edge-on galaxies. I have shown that self-consistent modelling can explain a number of problems: (i) the extension of soft X-ray halos, (ii) their spectra (although better photon statistics and higher spectral resolution are desirable), (iii) highly subsolar abundances in starburst galaxies are artifacts of the fit procedure, (iv) depending on the origin of the plasma there exist diagnostic ions that contribute to the emission in a characteristic fashion during the evolution of the outflow (along the minor axis in edge-on galaxies). Further studies will enable us to eventually connect the spectral behaviour to the star formation activity.

\section{References}

Avillez, M.A., Breitschwerdt, D., 2003, A\&A (in preparation)

Böhringer, H., Proc. of IAU Colloq. No. 166: The Local Bubble and Beyond, 1997, eds. D. Breitschwerdt, M.J. Freyberg, J. Trümper, Springer LNP 506, p. 341

Böhringer, H., Hensler, G., 1989, A\&A 215, 147

Breitschwerdt D., Freyberg, M.J., 2003, A\&A (in preparation)

Breitschwerdt D., Pietsch, W., Vogler, A., et al., 2003 (in preparation)

Breitschwerdt D., Schmutzler T. 1994, Nature 371, 774

Breitschwerdt D., Schmutzler T., 1999, A\&A 347, 650

Cox, D.P., Anderson, P.R., 1982, ApJ 253, 268

Heckman, T.M., Armus, L., Miley, G.K., 1990, ApJS 74, 833 
Masai, K., 1997, A\&A 324, 410

Raymond J.C., Smith B.W. 1977, ApJS 35, 419

Shapiro, P.R., Moore, R.T., 1976, ApJ 207, 460

Schmutzler, T., Tscharnuter, W.M. 1993, A\&A 273, 318 
breitschwerdt.tex; 29/10/2018; 13:07; p.12 